\title{
MULTILEVEL INVERTER FED INDUCTION MOTOR DRIVES
}

\author{
S Krishnapriya ${ }^{1}$, Unnikrishnan $\mathbf{L}^{2}$ \\ ${ }^{I}$ PG Scholar, Dept of EEE, Rajagiri School of Engineering and Technology, Kakkanad, Kerala,India, \\ krishnapriyas22@ymail.com \\ ${ }^{2}$ Asst. Professor, Dept of EEE, Rajagiri School of Engineering and Technology, Kakkanad, Kerala, India, \\ unnikrishnan4u@gmail.com
}

\begin{abstract}
Induction motor drive is used in almost all industries including oil and gas sectors, production plants and process industries. Speed control of induction motor is a major concern. The proposed technology implements a better speed control for induction motor drives using a multilevel inverter. The switching sequences are generated by optimized pre-calculated angles for controlling induction motor. And also with the use of a multi-level inverter there is lower distortion of output voltages and Total Harmonic Distortion (THD) of machine currents under steady state operating conditions can be minimized. THD can be further reduced by optimizing the switching angles. This technology can be extended to higher levels of output voltages by using an $n$ state multilevel inverter. In this paper, a seven level multilevel inverter is proposed, its simulation is done in MATLAB/Simulink and the results were verified by experimenting on a hardware prototype. This could be extended to higher levels of voltage waveform and can be used in photovoltaics. A multilevel inverter could be a better choice as a dc-dc converter in solar power circuits.
\end{abstract}

Keywords: Multilevel inverter, optimal pulse with modulation, total harmonic dlistortion.

\section{INTRODUCTION}

Induction motor is the most widely used electrical machine in almost all simple, medium and high voltage industrial applications, because of its low cost and increased reliability. Development of high power and low cost power electronic devices in the recent past has provided a larger area of application for the ac drives. Hence, ac drives like induction motor drives along with power electronic converters have replaced the dc motor drives in industries.

The difficulty in using ac drives lies with the selection of suitable power electronic converter. The non linear dynamic performance of the induction motor, which constitutes additional non-linearities in the converter dynamics and the switching performance, adds to the difficulty level of the control task. And also, when Pulse Width Modulation (PWM) is used for the control of the power electronic converter, duty ratio input needs to be bounded in a specific range as this can create stability issues. Thus, the power conversion stage is playing a vital role.

A multilevel inverter could be a great choice for replacing the conventional voltage source inverters or current source inverters. Multilevel inverters have many advantages like reduced voltage stress, increased quality of output voltage and increased power rating. Multilevel power converters can be defined as voltage synthesizers, in which many small discrete voltage levels add to synthesize a high output voltage as shown in Figure 1. The most attractive application of this technology is in the medium to high voltage range $(2-13 \mathrm{kV})$.

Jos Rodrguez et.al has explained about various multilevel inverter topologies and different modulation strategies in
[11]. Different multilevel inverter topologies are flying capacitor type, diode clamped and cascaded type inverters.

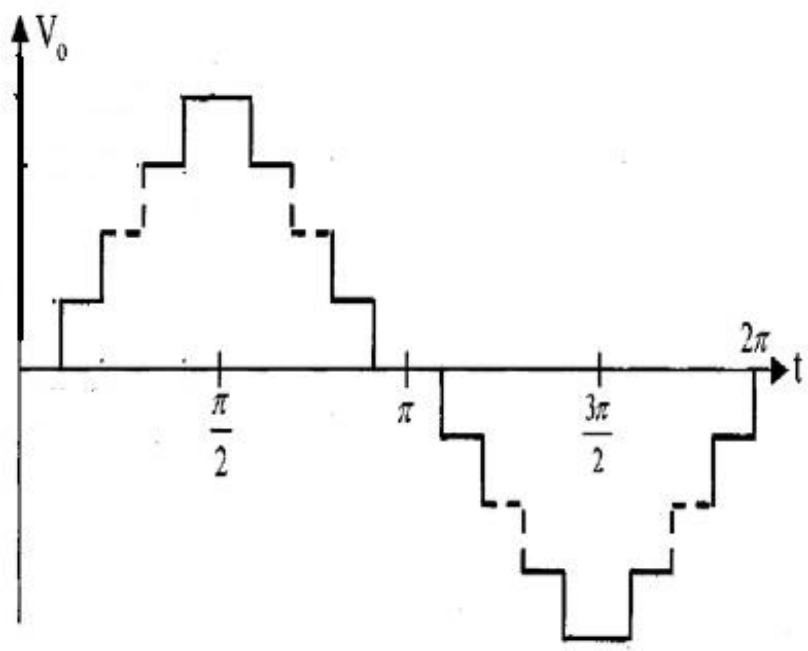

Fig 1: Generalised Stepped Voltage Waveform

Various modulation strategies used commonly are Sinusoidal Pulse Width Modulation (SPWM), Selective Harmonic Elimination (SHE), and Space Vector Control (SVC). Multilevel inverters can achieve higher voltage levels without the need of a transformer and also the voltage stress across the devices remains half, low dv/dt of the output voltage, and low electromagnetic interference, resulting in low Total Harmonic Distortion (THD). Thus, they are emerging as an attractive solution to mediumvoltage high-power ac drives. 
Akshay K. Rathore et.al have proposed a generalized optimized PWM technique (Synchronous Optimal PWM (SOP)) for higher levels of converters (L> 5) in [1] minimizes the harmonic distortion while reducing the switching frequency. It consists of optimizing switching angles to minimize THD of machine currents under steady state operating conditions.

Nashiren Farzilah Mailah et.al has explained on the calculation and determination of the triggering angle and switching duration for each switch with the aim of obtaining the lowest Total Harmonics Distortion output voltage waveform for a three phase 5 level inverter in [7]. The harmonics content decreased by using the neutral point clamped technology such that the need for filters can be avoided.

Use of artificial intelligence is gaining importance for harmonic elimination and optimization of PWM signals. Andres Fernando Lizcano Villamizar et.al has explained the use of the application of genetic algorithms in the optimization of the PWM modulation in [12]. This is done by creating a set of individuals (different values of frequency, pulse number and pulse position) representing possible solutions to the problem to be optimized and find the lowest harmonic distortion of the system, which is the fitness function (objective) to optimize the harmonic distortion.

Space Vector Modulation (SVM) technique is suitable for harmonic reduction when the switching frequency is $1 \mathrm{kHz}$ or higher. But, at low switching frequencies, it leads to unacceptable high harmonic distortion of the machine currents. SOP is one of the emerging techniques that permit the reduction of switching frequencies with less harmonic distortion. A generalized optimization algorithm for Synchronous Optimal Pulse width (SOP) modulation for a five level and three level inverter is explained in [8] by Akshay K. Rathore et.al.

\section{MULTILEVEL INVERTERS}

A multilevel power converter structure has been introduced as an alternative in high power and medium voltage situations where it is troublesome to connect a single power semiconductor switch. Subsequently, several multilevel converter topologies have been developed. A multilevel converter not only achieves high power ratings, but also enables the use of renewable energy sources.

Many multilevel converter topologies have been proposed during the last two decades. The most common among them are: cascaded H-bridges converter with separate dc sources, diode clamped (neutral-clamped), and flying capacitors (capacitor clamped). Moreover, various modulation techniques have also been developed. The overview of a modulation method is listed in Figure 1.

The most attractive features of multilevel inverters are as follows.

- They can generate output voltages with extremely low distortion and lower dv/dt.

- They draw input current with very low distortion.

- They generate smaller Common Mode (CM) voltage, thus reducing the stress in the motor bearings.

- They can operate with a lower switching frequency.

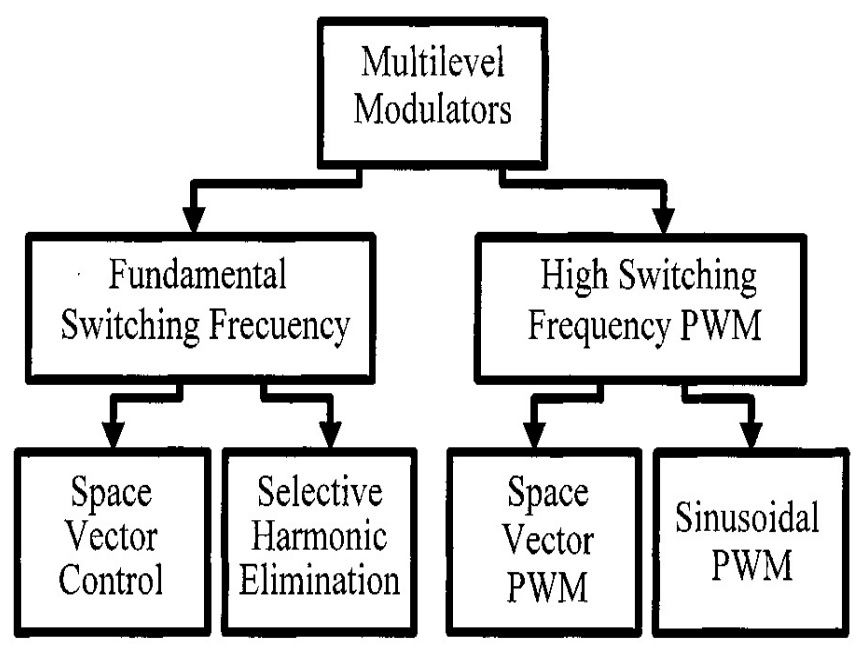

Fig 2: Classification of Modulation Strategies

Thus, a multilevel inverter can be used to control the induction motor with reduced harmonic distortion. A 5/3 H Bridge seven level inverter is proposed here as shown in Figure 3.

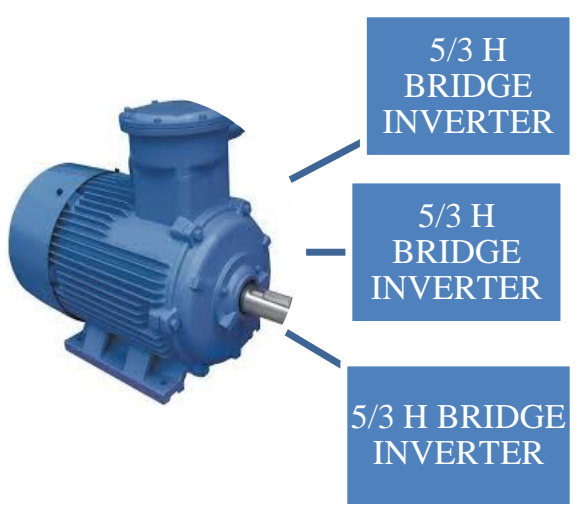

Fig 3: Proposed 5/3 h bridge inverter

\section{SYNCHRONOUS OPTIMAL PWM}

Synchronous Optimal Pulsewidth Modulation (SOP) is an emerging modulation technique that helps to reduce the harmonic distortion caused by the multilevel inverter. This method pre-calculates optimal switching angles in an offline procedure assuming steady state conditions, within a period of the fundamental period $1 / \mathrm{fs}$ to minimize the harmonic distortion. The switching frequency is synchronized with the fundamental frequency of the voltage waveform, therefore called SOP. This pattern or angles are programmed, or stored, as look-up tables in hardware. Since this is also synchronous modulation the number of pulses in positive and negative half cycle are equal. This method allows a considerable reduction of switching frequency without increasing the distortion. The pulse number, 


$$
N=\text { floor }\left(\frac{(L-1) f_{s, \max }}{2 f_{1}}\right)
$$

The number of switching instants (angles) over a quarter of the fundamental period should be an integer and hence command floor rounds the value within brackets to the nearest integer. $f_{s, \max }$ is the maximum switching frequency which restricts the dynamic losses of semiconductor devices, and $\mathrm{f} 1$ is the fundamental frequency of the voltage waveform. $\mathrm{L}$ is number of levels in the multi level inverter, i.e., $\mathrm{L}=3$ for three level inverter, $\mathrm{L}=5$ for five level inverter, etc. Pulse number increases with reduction in $\mathrm{f} 1$ as seen from equation (1).

The following conditions need to be satisfied while optimizing the switching angles over one fundamental period for each steady-state operating point:

- Minimum Distortion factor.

- Consecutive switching angles to be sufficiently separated to allow for minimum on times and off times of the power semiconductor switches

- Continuity of the switching angles within the modulation index range associated to a given pulse number.

The objective function for the offline optimization procedure is minimization of the distortion factor, $\mathrm{d}$ while calculating the switching angles. The objective function is therefore, Minimize:

$$
d=\frac{i_{h}}{i_{h, n-s t e p}}
$$

Where $i_{h}$ is the harmonic rms current and $i_{h, n-s t e p}$ is the harmonic rms current at n-step operation of the multilevel inverter.

\section{SIMULATION RESULTS}

A seven level inverter is designed by cascading a five level and three level $\mathrm{H}$ bridge inverter. Switching sequences are generated in an offline procedure as explained in [8] for the multilevel inverters. Simulation is done in MATLAB/Simulink software as in Figure 4. Subsystems in figure 4 are formed by cascading a three level inverter and five level inverter to give a seven level output voltage waveform. The output voltage is then filtered and fed to a three phase induction motor. The pre-calculated switching angles are generated using the controller. The circuit diagram for cascaded $5 / 3 \mathrm{H}$ bridge inverter is shown in figure 5 .

Figure 6 shows the speed and torque waveforms obtained after simulation. Figure 7 shows the output voltage waveform for a seven level multilevel inverter.

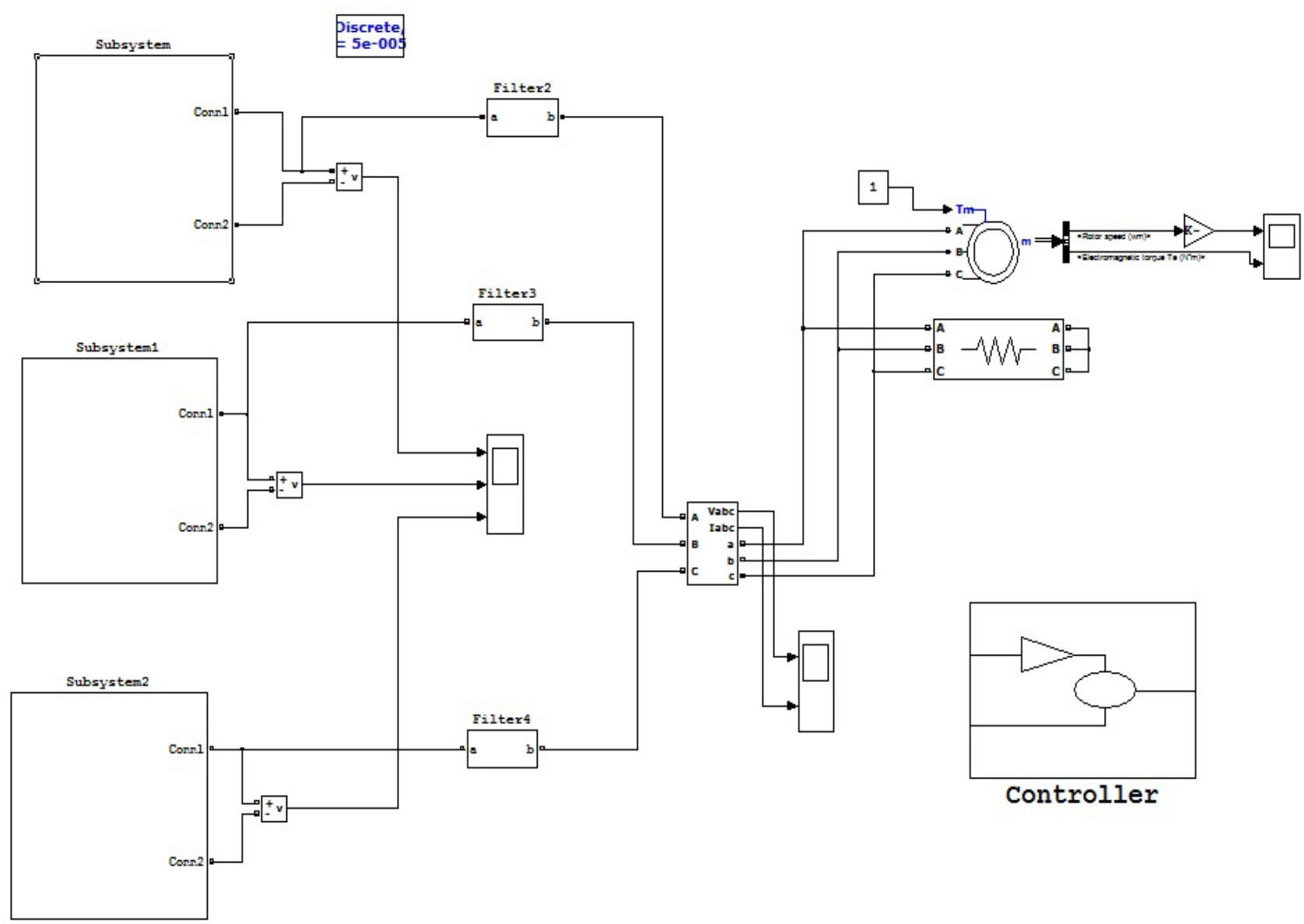

Fig 4: Simulation diagram for seven level inverter with motor 


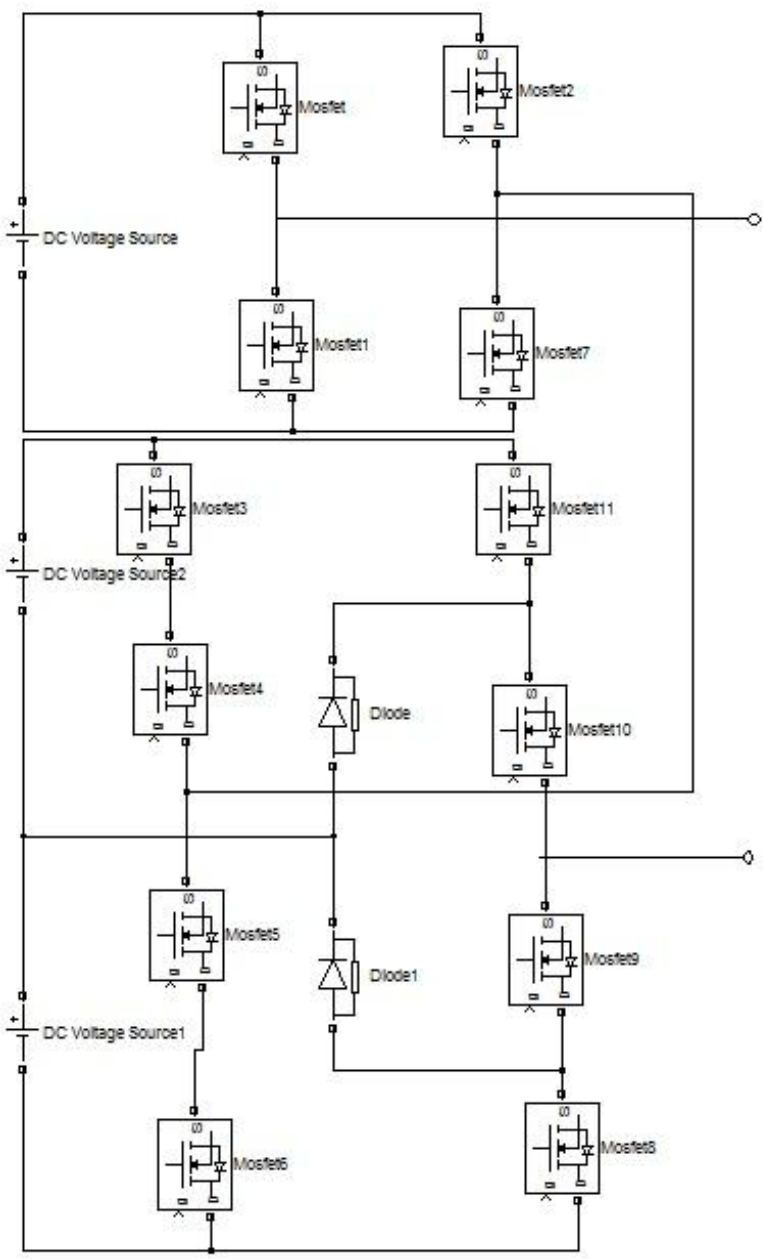

Fig 5: Cascaded 5/3 H Bridge inverter
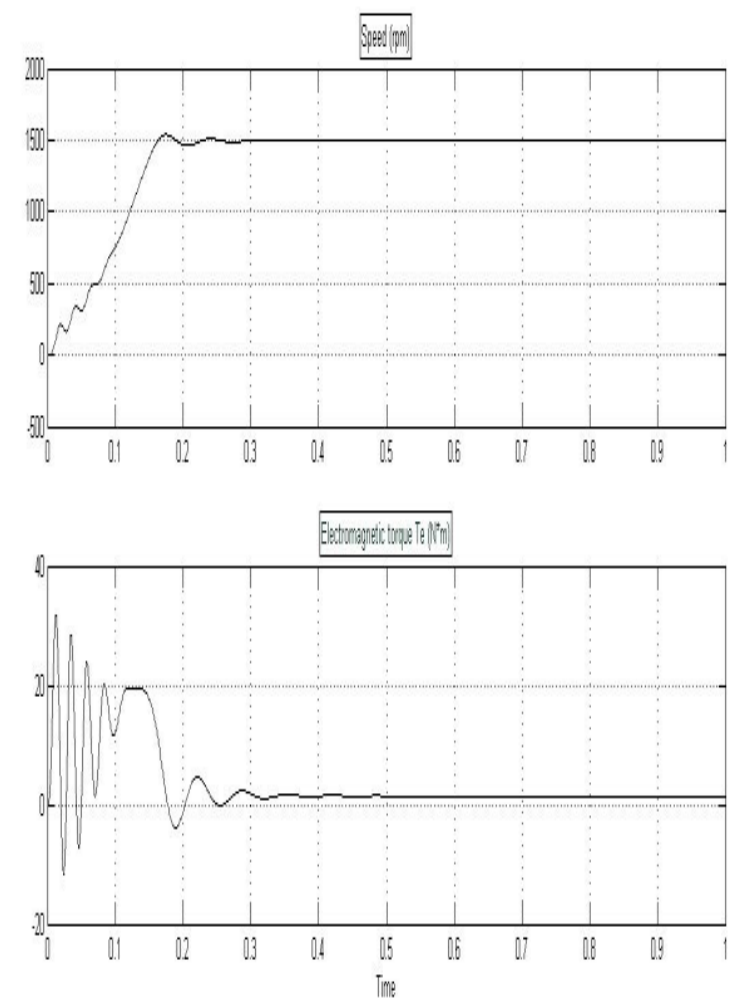

Fig 6: Torque and Speed Waveforms

\section{HARDWARE DESCRIPTION}

A prototype of the seven level inverter was developed using the PIC controller. The experimental setup and the results were shown in Fig 7 and 8.

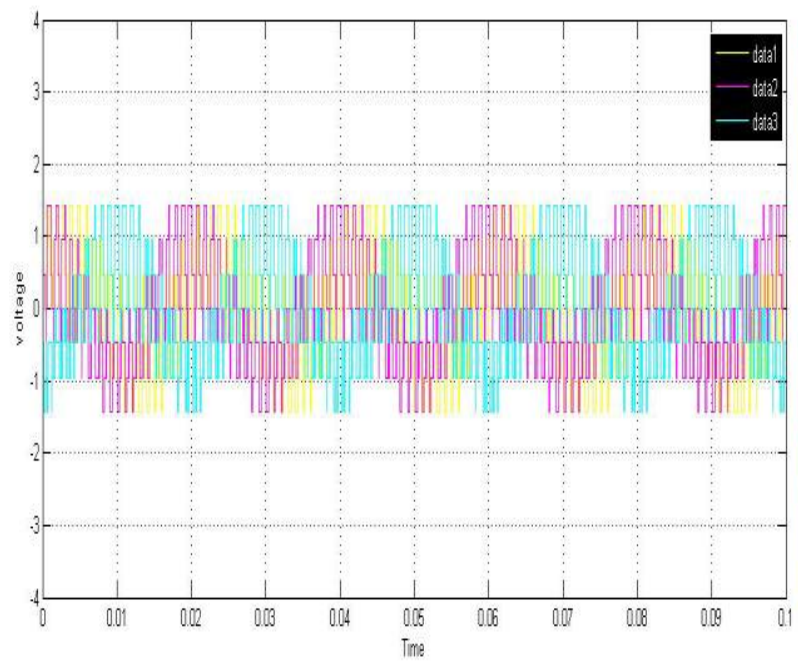

Fig 7: Output Voltage Waveforms

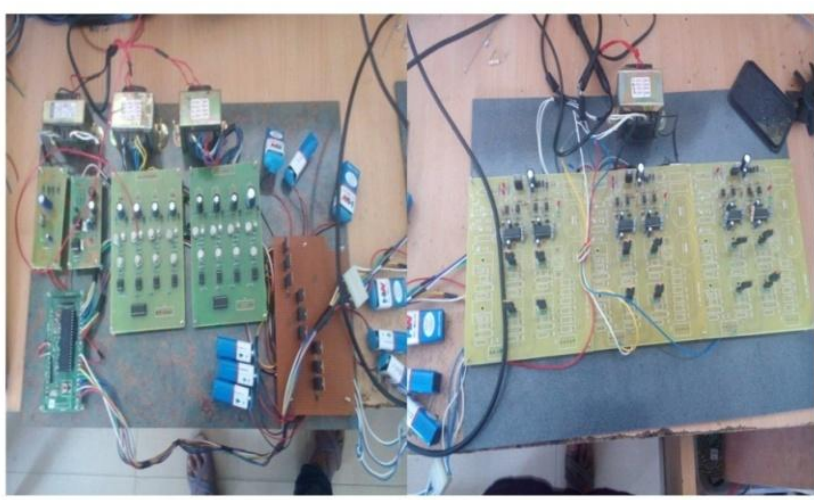

Fig 8: Experimental Setup

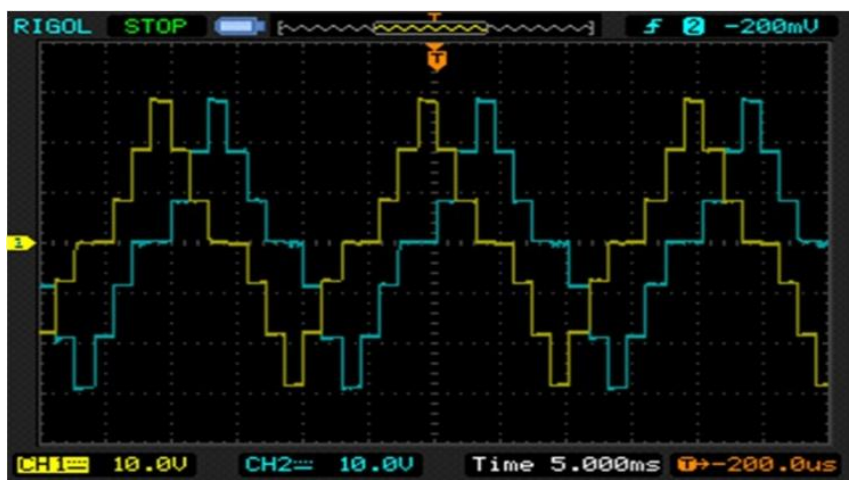

Fig 9: Seven level output from hardware

\section{CONCLUSION}

Induction motor is the most widely used machine in industries due to its various advantages. At present, Variable Frequency Drives (VFD) are used for its speed control. And disadvantage with this technology is the increasing THD. It has been studied that THD could be reduced by replacing a VFD with a multi level inverter. For this a seven level multilevel inverter is simulated in MATLAB and the results were studied. The switching sequences are generated using 
the precalculated switching angles in such a way to reduce THD. The topology can be applied to a higher number of levels of multilevel inerter. But, the disadvantage of using multilevel inverter is that with the increase in levels, switching circuit gets complicated.

\section{REFERENCES}

[1]. Amarendra Edpuganti and Akshay K. Rathore Optimal Low-Switching Frequency Pulsewidth Modulation of Medium Voltage Seven-Level Cascade$5 / 3 \mathrm{H}$ Inverter", IEEE Transactions on Power Electronics, vol.30, No.1, January 2015.

[2]. Kumar et al., "Seventeen-Level Inverter Formed by Cascading Flying Capacitor and Floating Capacitor HBridges",IEEE Transactions on Power Electronics, Vol.30, N0. 7, July 2015.

[3]. Alex Ruderman, "About Voltage Total Harmonic Distortion for Single- and Three-Phase Multilevel Inverters", IEEE Transactions on Industrial Electronics, Vol. 62,No. 3, March 2015.

[4]. Amarendra Edpuganti and Akshay K. RathorelOptimal Low Switching Frequency Pulsewidth Modulation of Nine-Level Cascade Inverter", IEEE Transactions on Power Electronics, Vol.30, No.1, January 2015.

[5]. Jackson Lago and Marcelo Lobo Heldwein, "Multilevel Synchronous Optimal Pulsewidth Modulation Generalized Formulation", Workshop on Control and Modeling for Power Electronics (COMPEL), 2014.

[6]. Till Boller, Joachim Holtz, and Akshay K. Rathore ,Weutral-Point Potential Balancing Using Synchronous Optimal Pulsewidth Modulation of Multilevel Inverters in Medium-Voltage High-Power AC Drives ",IEEE Transactions on Industry Applications, vol. 50, No. 1, January/February 2014.

[7]. Nashiren Farzilah Mailah et.al, "Harmonics Reduction of Three Phase Five-Level Neutral-Point-Clamped Multilevel Inverter", 2012 IEEE Conferenece on Power and Energy(PECon), Dec 2012.

[8]. Akshay K. Rathore et.al ,ISynchronous Optimal Pulsewidth Modulation for Low Switching-Frequency Control of Medium-Voltage Multilevel Inverters",IEEE Transactions on Industry Applications, vol. 57, No. 7, July 2010.

[9]. Bin Wu, Jorge Pontt, Jos Rodrguez, Ste_en Bernet and Samir Kouro "Current-Source Converter and Cycloconverter Topologies for Industrial MediumVoltage Drives", IEEE Transactions on Industrial Electronics, Vol. 55, No. 7, July 2008.

[10]. Jos Rodrguez et.al "Multilevel Voltage-SourceConverter Topologies for Industrial Medium-Voltage Drives", IEEE Transactions on Industrial Electronics, Vol. 54, No.6, December 2007.

[11]. Jos Rodrguez, Jih-Sheng Lai and Fang Zheng PenglMultilevel Inverters: A Survey of Topologies, Controls, and Applications", IEEE Transactions on Industrial Electronics, vol. 49, No. 4, August 2002.

[12]. Andres Fernando Lizcano Villamizar et.al, "The Application of Genetic Algorithms in Electrical Drives to Optimize the PWM Modulation ANDRS", Recent
Researches in Automatic Control, Systems Science and Communications.

[13]. R.Krishnan and P.Pillay, Modeling, simulation, and analysis of permanent-magnet motor drives II - The brushless DC motor drive", IEEE Trans. Ind. Applns.,vol. 25, no. 2, pp 274-279, 1989. 